\title{
PROSPECTIVA DE LAS COMPETENCIAS GERENCIALES EN LA GESTIÓN PÚBLICA*
}

\author{
Leoncio Solórzaño AcuÑa ${ }^{1}$ \\ Universidad Nacional Mayor de San Marcos, Lima, Perú \\ (RECIBIDO EL 21/10/2008, ACEPTADO EL 12/12/2008)
}

\begin{abstract}
RESUMEN
Más allá de las modas de gestión y de las mutaciones semánticas que enriquece la vida organizacional, la prospectiva permite comprender con cierta anticipación las probabilidades de alternativas de solución en la gestión pública. Actualmente, hay una discusión y comparación entre el antiguo y el nuevo paradigma de la gestión del Estado. Se describen los sistemas teóricos-conceptuales de los paradigmas del funcionario con relación a las competencias gerenciales.

La mayoría de los funcionarios públicos han sido entrenados y están ejerciendo sus labores en el Estado dentro de la antigua tradición del modelo burocrático y vertical, empantanándose en el conformismo, la rutina y la copia estéril, en desmedro de la creatividad y la innovación.

La nueva generación se está adecuando a la provocación de los nuevos paradigmas de la cultura organizacional con calidad humana y prospectiva. Es decir, es necesario que los trabajadores del conocimiento -servidores del Estado- deban modificar su comportamiento y asumir su responsabilidad desde el enfoque de las competencias gerenciales. Finalmente, se propone algunas sugerencias para que los funcionarios hagan trabajar todo su cerebro dentro de lo deseable, lo probable y lo posible de la gestión pública y promover una Política de Estado competitiva y productiva.
\end{abstract}

Palabras clave: Competencias gerenciales. Funcionario Público. Gestión pública. Liderazgo de gestión. Paradigmas. Prospectiva.

\begin{abstract}
Beyond the fashions of management and the semantic mutations that enriches the organizationallife. The prospective allows to understand with certain anticipation the probabilities of alternatives of solutions in public administration. Currently there is a discussion and comparison between the old and the new paradigm of the management of the State. It describes the systems of theoretical and conceptual paradigms of public officials with regard to managerial skills.
\end{abstract}

\footnotetext{
* Ponencia presentada en el IV Congreso Nacional Prospecto Perú. Trujillo, setiembre de 2008.

1 Docente Principal de la Facultad de Psicología de la UNMSM. E-mail: Isolorzañoa@unmsm.edu.pe
} 
Most civil servants have been trained and are exercising their work in the State within the old tradition of the bureaucratic and vertical model, being swamped in the routine, conformism and sterile copying, in impairment of the creativity and the innovation.

The new generation is being adapted to the provocation of the new paradigms of the organizational culture with prospective and human quality. That is, it is necessary for public servants to modify their behavior and assume with responsibility the approach of management skills. Finally, it is being proposed some suggestions for public officials to work with all their brain within the desirable, probable and possible in the public administration and promote a State policy that is competitive and productive.

Palabras clave: Competencias gerenciales. Funcionario Público. Gestión pública. Liderazgo de gestión. Paradigmas. Prospectiva.

\section{INTRODUCCIÓN}

"La Gerencia de Conocimiento no es un producto de software o una categoría de software, ni siquiera comienza con tecnología. Comienza con los objetivos y procesos del negocio y con el reconocimiento de la necesidad de compartir información... No es más que administrar los flujos de información para dar la información correcta a la gente que la necesita, de tal manera que pueda usarla rápidamente".

Bill Gates

El funcionario del Estado debe tener las condiciones para encontrar la información, compartirla y utilizarla en su trabajo diario. El lograr esto implica cambiar muchas creencias arraigadas en la mente de los "funcionarios jefes", tales como: "Esta información es confidencial", "Tiene usted autorización para ver este informe?”, "Está prohibido accesar a internet o descargar de internet ciertos tipos de archivos en horas de oficina”, "póngalo en la intranet, pero protéjalo con un password para que solamente mi gente y yo podamos accesarlo", y otras que todos hemos escuchado. Entonces, hay que erradicar las actitudes inadecuadas y generar políticas de cambio de uso de información y conocimiento.

En la actualidad, las organizaciones de la sociedad del conocimiento están utilizando la prospectiva como herramienta estratégica para construir su visión, misión y objetivos estratégicos. Es decir, la prospectiva no es profecía, ni predicción, porque no tiene por objeto predecir el futuro, sino ayudarnos a construir y nos invita pues a considerar el futuro como algo por hacer, por construir, en vez de verlo como algo que ya estaría decidido, y del que sólo faltaría descubrir el misterio. Hugues de Jouvenel (2007) dice "La prospectiva es la hija de la libertad y de la responsabilidad. No es pues, un oficio, menos una caja de herramientas. Es más bien una filosofía, una forma de ser, una manera de ver las cosas, y, para aquellos que la practican, a veces sin saberlo, es un modo de vida".

Las fronteras se están transformando de barreras en paredes celulares de un organismo mayor, sin que esto signifique abdicar de la propia idiosincrasia cultural. Los Estados están abandonando los obsoletos, costosos e infructuosos esquemas tradicionales; en cambio están adoptando los nuevos esquemas estratégicos, para ser un Estado moderno, pequeño, fuerte, desconcentrado y eficiente. Estamos en una "cultura informática", que genera una 
inmensa cantidad de nuevos conocimientos y tecnologías promoviendo nuevos paradigmas de libertad de gestión con rostro humaño.

Los paradigmas son modelos conceptuales, patrones de conducta, conjunto de ideas o situaciones que facilitan a cómo resolver los problemas en la realidad. Los paradigmas influyen en todo nivel y en todo tiempo que dan origen a las actitudes y conductas para comprender y explicar ciertos aspectos de la realidad, a fin de obtener resultados.

El cambio de paradigma es un quiebre hacia un nuevo juego, un nuevo conjunto de reglas y un nuevo modo de hacer las cosas. En sí, se rompen por necesidad o se cambia por otro, generando resistencia, a veces en los implicados. En tal sentido, existen afirmaciones respecto a los paradigmas.

\section{Antiguos paradigmas}

Para algunos los paradigmas son la "regla de oro" para cumplir con sus objetivos, naturalmente con sus limitaciones. Los antiguos paradigmas pueden ser obstáculos en la gestión pública:

- $\quad$ Dificultad de percibir las nuevas oportunidades. El observar un hecho desde una sola perspectiva es un error que puede hacer de la vida cotidiana una desgracia.

- Minimiza la trascendencia de los hechos. Saber definir en su verdadera y justa dimensión los antiguos paradigmas que obstaculizan metas, atribuciones, creencias o enfoques.

- No facilita buscar información adecuada. Los mapas mentales tradicionales te anclan en el pasado y no te permiten interpretar lo que observas o experimentas, en otras palabras, te eclipsan.

- Distrae recursos en hechos irrelevantes. Los antiguos paradigmas, casi siempre conducen a la rutina y bloquean la observación de hechos interesantes,

- Interfiere en la creatividad. Los antiguos paradigmas casi siempre son barreras para la creatividad y no facilitan el cambio.

- No facilita la discriminación adecuada. Los paradigmas tiene repercusiones en la discriminación para percibir la conducta actual y ver el futuro con objetividad.

\section{Paradigmas del funcionario público}

Mientras transcurre la primera década del siglo XXI, estamos atravesando cambios muy profundos en el modo de ver la realidad, y ello, también abarca a las organizaciones. Lo cierto es que hay señales indudables de que se puede ver la realidad de otro modo.

\section{Funcionario del ayer}

En algunas personas el pasado es el gran referente traductor y distribuidor de significados. Hay barreras entre la persona creativa y la burocracia administrativa. Alguien dijo: "No mantener vivo a los muertos, sino dejar que nazcan los vivos". 
- $\quad$ El jefe paga mi sueldo y me debo a él. Hay que mantener contento y feliz al jefe, cumpliendo fielmente sin duda ni murmuraciones. Por ejemplo, "estando con Dios los santos son inquilinos".

- Una "pieza" más en la organización. El funcionario se considera un objeto sin iniciativa y cumple los "reglamentos o normas". Por ejemplo, "Si Ud. ordena mi jefe si se puede".

- Inteligencia racional. La mucha importancia al coeficiente intelectual (IQ) basado en la lógica y la razón, ha generado egoísmo, individualismo, entre otros.

- Todo tiempo pasado fue mejor. Muchos razonamos con creencias, conceptos y métodos superados hace décadas. Por ejemplo, "más vale lo malo conocido, que lo bueno por conocer".

- Mañana será igual que hoy. La mayoría no está preparado para razonar en posibilidades de éxitos en el futuro. Por ejemplo, "que viva la jarana aunque no se coma mañana".

\section{Funcionario público de hoy}

La velocidad en el cambio organizacional no da tiempo para administrar el conocimiento con los métodos tradicionales. Ahora las personas quieren ser protagonistas y líderes de gestión.

- Inteligencia emocional. La inteligencia aplicada a las emociones facilita reconocer, motivar y manejar consigo mismo y las relaciones con los demás.

- Políticos pagan mi sueldo. Hay que ser fiel a los políticos de turno para conseguir asuntos personales, no es necesario preocuparte de los usuarios.

- Pieza importante en la organización. El funcionario es pionero participando con iniciativas en el mejoramiento del servicio o producto dentro de la organización.

- Nadie sabe como será mañana. Visionario y está preparado para las nuevas contingencias que podría venir en el futuro. Por ejemplo, "Siempre listos" como los Boys Scout.

\section{Funcionario público del mañana}

Cuando cambian los paradigmas, el mundo mismo cambia con ellos, lo cual implica incluso un cambio en la percepción de los fenómenos. En este contexto, se observa todavía:

- La comunidad paga mi sueldo. Evidenciar eficiencia y eficacia en el servicio a la comunidad, porque ellos determinarán quienes se quedan o quienes se van por deficientes.

- Innovar y crear. La creatividad es un proceso deliberado destinado a buscar y favorecer el desarrollo de resultados originales y novedosos. 
- Mayor competitividad y menores márgenes de error. Los funcionarios sin ser dioses, ni titanes, podemos hacer brotar nuevas ramas del árbol y hacer surgir la vida en medio del desierto.

- Organización por procesos, flexibles y horizontales. Estas se adaptan a los nuevos cambios de la organización. La estructura piramidal y funcional genera pérdida de tiempo.

- Inteligencias múltiples. Gardner (2003) propone 8 tipos de inteligencias: Intrapersonal, interpersonal, lingüística, visual-espacial, corporal, lógico-matemático, musical y naturalista.

\section{Competencias}

No sirven las excusas, sino la creación de valor que debe lograrse cuando las capacidades distintivas o competitivas esenciales se aplica en el ambiente correcto que las sepan apreciar y remunerar apropiadamente (Calderón, 2004). En este ámbito se define las competencias:

- Desempeño complejo que permite a la persona interactuar eficazmente en un medio.

- Capacidad para responder con éxito una demanda compleja o tarea.

- Una combinación dinámica integrada de conocimiento, habilidad, actitud y conducta para llevar a cabo una misión compleja en la comunidad y el Estado.

- Los resultados de aprendizaje de un programa educativo o lo que los alumnos son capaces de demostrar al final en su proceso formativo.

- Característica subyacente en un profesional que está causalmente relacionadas con un desempeño excelente en la comunidad.

\section{Gerencia}

La gerencia tiene como piedra angular al ser humaño, quien es el cliente, usuario, trabajador, miembro de una comunidad, integrante de un Estado, etc. Entonces, gerenciar es lograr resultados mediante la acción de otras personas a las que se les proporciona el ambiente propicio para la toma de decisiones en forma oportuna y correcta (E. Valdez, 1998). Es decir, es persuadir y mentalizar con flexibilidad y ética en todos los niveles ejecutivos y en los demás trabajadores para que acepten los modelos propuestos de comportamiento, creencias y valores de la organización.

\section{Competencias gerenciales}

Las competencias son las características personales -conocimiento, habilidades y actitudes- que llevan a desempeños adaptativos en ambientes saludables. Asimismo, existen competencias subjetivas que corresponden a la apreciación interna del sujeto de sus 
competencias: concepto de sí mismo, autoestima, autoeficacia, responsabilidad, expectativa de resultados y autoconfianza.

Las competencias gerenciales son aquellas que facilitan la forma cómo hacer las cosas para solucionar problemas y tomar decisiones dentro del proceso del desarrollo organizacional. Aquí es importante la asimilación y la acomodación de conocimientos, métodos, técnicas y procedimientos de manera informal y formal, especialmente en situaciones de incertidumbre.

El gerenciar es persuadir y mentalizar a los niveles ejecutivos y trabajadores para que acepten una organización flexible y horizontal. Esto lleva a la creación de redes humanas virtuales, donde la organización será capaz de autodesarrollarse basándose en la maximización interna, metódica y sistémica de sus integrantes para asimilar el cambio e interpretar los mensajes de los usuarios.

Los gerentes líderes asimilan los aportes de la Administración Moderna para aplicarlos en la gestión pública. En este contexto, visualizamos los modelos teóricos y las competencias gerenciales:

\section{Modelo conductual}

La función está basada de acuerdo a los criterios de la eficacia de la conducta observable del funcionario, a través de la productividad y los beneficios de los medios-fines en una dirección clara que lleva a resultados. Asume una conducta severa, estable y de liderazgo, clarificando las metas, el análisis racional y la acción de logros. La tarea es actuar en las competencias o roles como:

- Director: sabe conducir. Clarifica las expectativas de la planificación y fijación de objetivos; define los problemas, roles y tareas; selecciona objetivos y alternativas de solución. Un buen director es un gerente que: Demuestra que las ideas tienen valor, respeto profundo a soluciones novedosas, no le teme al fracaso, es eterno aprendiz, revisa las actividades originales y ejecuta nuevas formas de conductas positivas, orienta los contactos con el medio ambiente.

- Productor: sabe hacer. Tiene elevada motivación, profesionalismo, responsabilidad centrado en el trabajo y los objetivos organizacionales. El productor promueve: el Potencial humaño, basado en la motivación personal para la ejecución de tareas y metas; el sistema productivo que administra recursos humaños y materiales para lograr bienes y servicios de calidad reconocida; y el medio ambiente que debe ser respetado para las buenas prácticas de vida saludable.

- Competitivo: Saber hacer. Mantiene ventajas comparativas que le permitan alcanzar, sostener y mejorar una posición en el entorno de permanente cambio. Se compromete a la acción y convierte a sus seguidores en líderes de cambio, a través de: accesibilidad al servicio vía costo, calidad, diseño, metodología, etc.; grado de aceptación de los productos y servicios a nivel nacional e internacional; y logros empresarial determinados por sus circunstancias específicas. 


\section{Modelo cognitivo}

Los criterios están determinados por los procesos del capital intelectual: definición de responsabilidades, evaluación con criterios, manejo de documentación y el mantenimiento del registro de la información. El cognitivo es jerárquico y las decisiones están matizadas por las normas, estructuras y modelos existentes. En este modelo se debe tener en cuenta las competencias como:

- Monitor: sabe orientar. Evidencia preferencia por los trabajos administrativos, por buscar y facilitar información rutinaria, realizar inspecciones, visitas y autoriza la revisión de los trabajos y demás tareas que le permita superar dificultades: "Sabe qué es lo que está sucediendo".

- Coordinador: sabe integrar: Posee una autoimagen positiva y equilibrada. El coordinador hace uso adecuado del conocimiento y constituye una influencia clave sobre la motivación y la cultura organizacional. Para ello, recurre a las funciones: Planeamiento, organización y control.

- Investigador: sabe explicar el porqué. Los propósitos son la investigación básica, aplicada y tecnológica. Es decir, evidenciar conducta científica para describir, explicar, predecir y experimentar los fenómenos o hechos de la realidad dentro de su ejercicio profesional.

\section{Modelo humanista}

El humanismo es un proceso central en una organización para la participación, la resolución de conflictos y la creación del consenso con clima de fraternidad. La tarea es ser:

- Facilitador: Sabe dar. Trato sencillo y noble en la docencia. Sabe que sus éxitos se los debe a otras personas. El profesional de calidad están dotado de las cualidades siguientes: crear equipos de trabajo para la competitividad; toma de decisiones participativa; y gestionar los conflictos.

- Mentor: Sabe guiar. Participa en el desarrollo personal de los demás. Apoya las peticiones legítimas, reconoce los méritos y planifica el crecimiento de sus subordinados, a través: comprensión incondicional a los demás, confianza en la comunicación interpersonal; y facilita el desarrollo de los trabajadores con retroalimentación proactiva.

- Marketing: Sabe "vender" imagen. Lograr mantener y aumentar el número de adeptos mediante la creación y entrega de información o conocimiento con valor añadido superior a lo esperado por el usuario. En este ámbito se requiere ciertas cualidades: marca distintiva personal para superar a sus competidores; profesionalismo comprometido con el verdadero desarrollo; y genérico para movilizarse con responsabilidad en la laboriosidad organizativa.

\section{Modelo estratégico}

Aquí la organización pone énfasis en los criterios propios de un pensamiento abierto y libre para buscar nuevas formas de hacer las cosas y siempre se están preguntados, los porqués. Estas personas tienen una visión nueva para ponerlas en práctica. La tarea directiva en este modelo es: 
- Innovador: hace novedades. Consiste en aportar algo aún desconocido para mejorar un producto, proceso o servicio. Podemos hablar de innovación: social que propone soluciones nuevas a los problemas sin trastocar la eficiencia de la organización; tecnológica condición fundamental de un producto o proceso exitoso en el mercado; y gestión que facilita el manejo adecuado de los recursos disponibles con valor agregado tanto social como tecnológico.

- $\quad$ Broker. Sabe negociar. Negociación proceso informal y flexible que se lleva a cabo sin la intervención de un tercero para la búsqueda de un acuerdo consensual "frente afrente" entre dos o más partes en conflicto y lograr la satisfacción de sus respectivos intereses; mediación, proceso informal de carácter privado, confidencial y flexible de negociación facilitada por un tercero neutral, el cual no tiene la facultad de proponer opciones de solución al conflicto; conciliación, proceso de negociación asistida por un tercero denominado conciliador, que ayuda a que las partes encuentren una solución consensual que satisfaga sus intereses y es de estricto y obligatorio cumplimiento.

- Caoching (Hacer exitoso). Herramienta estratégica para el alto desempeño exitoso. Es un trabajo directo con un colaborador para mejorar una habilidad o comportamiento relacionado con su trabajo o su desempeño como un todo. En sí, es el arte de conseguir que tu colaborador se declare voluntariamente "culpable" y que diseñe el plan de mejora con indicadores de medición y revisión de desempeño. Así tenemos: coaching directivo uno con intervención personalizada para el directivo, no existe relación de autoridad; coaching de equipo donde se evidencia entre el líder y equipo para mejorar su desempeño y puede existir relación de autoridad; y coaching de desempeño, aquí si existe relación de autoridad, el jefe busca mejorar el desempeño del colaborador y que haga suyos los objetivos de aprendizaje y mejora para lograr resultados.

\section{Competencias gerenciales del funcionario público}

\section{FLEXIBILIDAD}

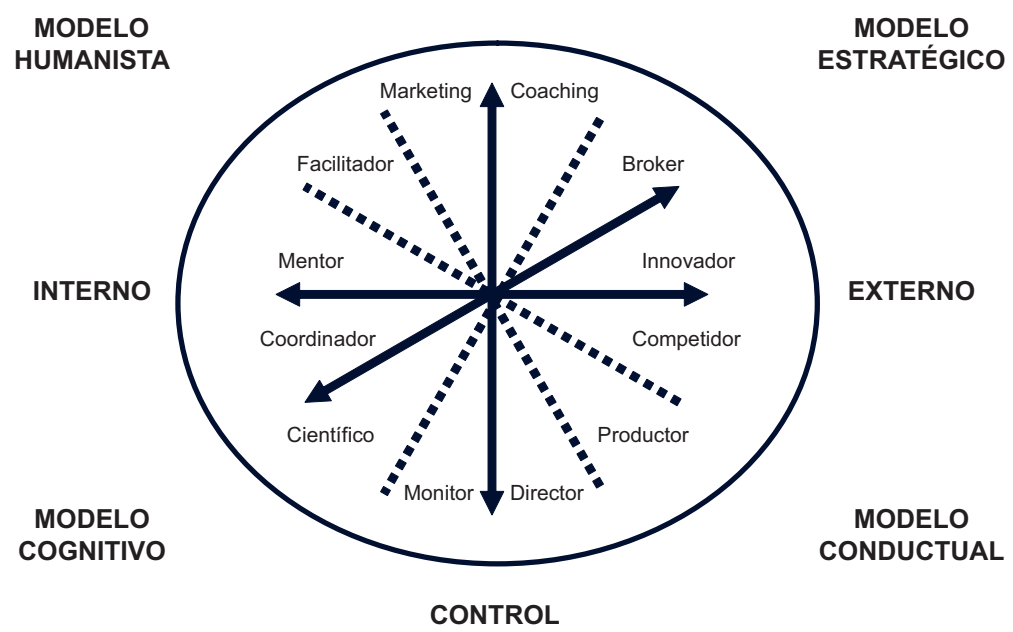

Fuente: Enrique Cárdenas (2004) “Competencias Gerenciales”. Universidad ESAN 


\section{Competencias gerenciales en la gestión pública}

El Estado es una sociedad política autónoma y organizada para estructurar la convivencia, en razón de que se trata de un conjunto permanente de personas que se relacionan por la necesidad de satisfacer imperativos afines de supervivencia y progreso común (García, 2008). Para ello se requiere de un sistema de relaciones coexistenciales dotado de fuerza social y basado en una relación humana saludable, "no hagas a otro lo que no quieres que hagan contigo": gobernantes y gobernados.

En el futuro es factible construir estrategias alternativas que garanticen un Estado deseable, probable y posible. Aquí intervienen las mentes creativas para obtener logros o resultados del caos, de la crisis y de la incertidumbre. Las personas trascendentes facilitan anticiparse y predecir, el impacto de los nuevos valores, nuevos conceptos y nuevas competencias gerenciales.

Estamos en la sociedad del conocimiento caracterizado por la creciente importancia del conocimiento recurso clave en la productividad y competitividad; la intensificación de la velocidad en tiempo real que producen cambios inesperados; la revolución tecnológica de las comunicaciones que promueven la mayor facilidad a problemas empresariales; y la globalización que está penetrando hasta lo más íntimo de las personas.

La gestión pública de calidad se propaga a la velocidad de la luz en la administración del Estado y existen algunas definiciones al respecto:

- Conjunto de lineamientos de comportamiento en la organización, planificación, dirección y control de una red de personas imbuida en la cultura organizacional que con apoyo de la tecnología de la información crea, renueva, organiza, trasmite, usa y protege los conocimientos tangibles e intangibles para satisfacer más allá de las expectativas de la comunidad y el Estado.

- Capacidad para crear nuevos conocimientos, saber compartirlos en forma adecuada, proteger ese conocimiento de los competidores y saber desechar el conocimiento desactualizado; sin obviar la tecnología, base de datos y herramientas de extracción de informaciones sistematizadas.

Por lo tanto, la gestión de pública es la capacidad de organizar un "círculo virtuoso" y está potencialmente latente en todos los individuos sin excepción y sólo necesita priorizar el uso del pensamiento aventurero con nuevos esquemas mentales y nuevas experiencias. Sin embargo, todavía muchos somos expertos en el pasado y tenemos la costumbre de "mantener vivo a los muertos e impedir que los vivos nazca" (De Bono E.,1995). Para generar credibilidad, confianza y proactividad en la comunidad y el Estado, sería pertinente tener en consideración algunas sugerencias en el marco de la realidad nacional:

\section{Gestión pública competitiva}

Hoy tenemos una oportunidad extraordinaria en el desarrollo organizacional de que los recursos claves, no son ya esencialmente físicos ni financieros como en el tiempo de la revolución industrial, hoy en día son intangibles, y depende en particular, de la capacidad 
de movilizar el capital intelectual, a través de la creatividad humana. André-Yves Portnoff (2008) considera: "La innovación es la aplicación de una idea conducida hasta su explotación efectiva en la sociedad, entonces, es el resultado concreto de una idea que logra su objetivo porque corresponde a dos campos de posibilidades: el científico y el tecnológico por una parte, y el social por otra parte”.

El futuro deseable es factible construir y definirlo proactivamente para mejorar los estilos de vida en comunidad y proponer alternativas de solución a los conflictos sociales. Las organizaciones están inmersas en el desafío de las nuevas modalidades de productos y servicios con alta rentabilidad donde prima lo intangible.

La gestión pública debe ser un conjunto de análisis y estudios prospectivo con el fin de explorar o predecir el futuro mediante el empleo de determinados métodos y herramientas para la consecución de los objetivos de la Política de Estado. En sí, es estar animada de cierta capacidad de cambio en tiempo real para diseñar "lo que deseamos que pase" y contribuir con efectividad en el desarrollo sostenible de la sociedad peruana, a través:

- Lo deseable. Aquí es muy importante tener en consideración la responsabilidad ética en los nuevos escenarios sociales, económicos, político-jurídicos, tecnológicos y ecológicos. Si es posible solo exigir a todos los trabajadores del Estado, el cumplimiento del Código de Conducta para Funcionarios con rostro humaño.

- Lo probable. Promover estrategias para mejorar la calidad humana dentro de la cultura organizacional donde se priorice: Autoestima como una necesidad psicológica de alta valoración de sí mismo y se sientan orgullos de ser el capital intelectual del Estado; asertividad que facilite la comunicación interpersonal sana y madura donde se respete los derechos personales y la dignidad humana; y toma de decisiones que deben evidenciar saber utilizar todo el cerebro individual o corporativamente para generar estrategias de acciones oportunas en situaciones de incertidumbre.

- $\quad$ Lo posible. La persona autorrealizada tiene la opción de contribuir con su creatividad e innovación en el encuentro corporativo de la cultura organizacional donde tiene presente: Relaciones humanas saludable que puede ser expresado en forma sencilla y franca: "No hagas a otro lo que no quieras que hagan contigo"; trabajo en equipo donde cada integrante tenga la posibilidad de aprender a saber vivir en equipo, comprometerse en la sinergia y generar una cultura sabedores exclusivos/inclusivos en beneficio de los demás; y liderazgos en situaciones caóticas, aquí es importante demostrar coraje, intuición y tiempo en la conducción de sus seguidores con visión positiva de futuro, saber utilizar el poder para servir y ser un eterno estudiante.

\section{REFLEXIONES FINALES}

El Estado tiene la necesidad de exigir la formación de profesionales de clase mundial o cosmopolita a su servicio, en base a tres activos intangibles denominadas las tres ces $(3 \mathrm{C})$ : conceptos, pensar creativamente y tener un marco conceptual científico y tecnológicos en su competencia profesional; competencias, saber hacer las cosas bien con innovación y cero defectos, entregando un servicio, proceso o producto más allá de las expectativas del usuario; 
y contactos, demostrar compromiso sincero y solidaria para granjearse de verdaderos amigos, ser facilitador estratégico en el trabajo en equipo y promotor de los líderes estratégicos.

La psicología debe involucrase en la gestión pública para darle rostro humaño o calidad humana a las competencias gerenciales. El funcionario público debe captar, crear, innovar y gerenciar el conocimiento; y que las competencias gerenciales son herramientas de gestión para éxito de las organizaciones.

La prospectiva requiere que cada uno de nosotros como funcionario público le dé a la imaginación alas con metas claras y armonizar con responsabilidad social entre: saber saber, saber hacer, saber ser, y poder hacer. Para modernizar una Estado o una organización recordar que las "leyes, normas y reglamentos, es corral para los animales y un punto de referencia para la gente inteligente" etc. Por lo tanto, hay que cambiarla de actitud de la gente, porque la gente hace la diferencia; a través de un sistema educativo que promueva pioneros de la creatividad y la innovación; sólo así podemos tener profesionales que demuestren competencias gerenciales en el manejo de la cosa pública con calidad y sensibilidad social.

"No existe un camino libre para el futuro. No hay autopistas pavimentadas desde el hoy hasta el mañana. Solo hay desiertos. Solo terreno incierto. No hay mapas de carreteras. No hay señales en la vía. Entonces, promover líderes que se confíen a la brújula creativa y al sueño despierto".

Robert Swiggett

\section{REFERENCIAS BIBLIOGRÁFICAS}

1. BARKER, Joel (2000). Separata Paradigmas. Lima: Universidad de Lima.

2. CALDERÓN, Luis (2004). Después de todo, sólo somos seres humaños. Lima: Ed. Business, ESAN. Lima.

3. COVEY, Stephen (1998). Los 7 hábitos de la gente altamente efectiva. Buenos Aires: Ed. Paidós, Bs. Aires.

4. GARCÍA TOMA, Víctor (2008). Teoría del estado y derecho constitucional. Lima: Ed. Palestra.

5. DE JOUVENEL Hugues (2007). Invitación a la prospectiva. Lima: Ed. CONCYTEC.

6. MENDOCA, Sebastiao (1998). La creación de futuros. Lima: Ed.Visual Service S.R.L.

7. MORRIS, Eddie (2006). Planeamiento estratégico de desarrollo personal. INAEP.

8. MOSS, Rosabeth (1995). Directiva de clase mundial. México: Ed. Interamericana.

9. ORTEGA, Fernando (2008). Prospectiva en seguridad ciudadana. Separata, INAEP

10. PORTNOFF André-Yves (2008). Por la senda de la innovación. Lima: Ed. CONCYTEC. 
11. ROBBINS, Stephen (2002). Comportamiento organizacional. México: Ed. Prentice Hall.

12. SOLÓRZAño ACUÑA, Jorge Leoncio (2007). El portal de paradigmas para organizaciones competitivas I (Lo deseable): "La responsabilidad ética en los nuevos escenarios sociales”. Lima: Ed. UNMSM.

13. SOLÓRZAño ACUÑA, Jorge Leoncio (2007). El portal de paradigmas para organizaciones competitivas II (Lo probable): "Estrategias para mejorar la calidad humana”. Lima: Ed. UNMSM.

14. SOLÓRZAño ACUÑA, Jorge Leoncio (2007). El portal de paradigmas para organizaciones competitivas III (Lo posible): "De la realización personal al encuentro corporativo”. Lima: Ed. UNMSM. 